\title{
Evaluating in vivo efficacy - toxicity profile of TEG001 in humanized mice xenografts against primary human AML disease and healthy hematopoietic cells
}

Inez Johanna ${ }^{1 \dagger}$, Trudy Straetemans ${ }^{1 \dagger}$, Sabine Heijhuurs ${ }^{1}$, Tineke Aarts-Riemens ${ }^{1}$, Håkan Norell ${ }^{2}$, Laura Bongiovanni ${ }^{3}$, Alain de Bruin ${ }^{3}$, Zsolt Sebestyen $^{1+}$ and Jürgen Kuball ${ }^{1 *+}$

\begin{abstract}
Background: $\gamma 9 \delta 2 T$ cells, which express $V Y 9$ and $V \delta 2$ chains of the $T$ cell receptor (TCR), mediate cancer immune surveillance by sensing early metabolic changes in malignant leukemic blast and not their healthy hematopoietic stem counterparts via the y982TCR targeting joined conformational and spatial changes of CD277 at the cell membrane (CD277J). This concept led to the development of next generation CAR-T cells, so-called TEGs: aßT cells Engineered to express a defined $\gamma \delta T C R$. The high affinity $Y 9 \delta 2 T C R$ clone 5 has recently been selected within the TEG format as a clinical candidate (TEG001). However, exploring safety and efficacy against a target, which reflects an early metabolic change in tumor cells, remains challenging given the lack of appropriate tools. Therefore, we tested whether TEG001 is able to eliminate established leukemia in a primary disease model, without harming other parts of the healthy hematopoiesis in vivo.
\end{abstract}

Methods: Separate sets of NSG mice were respectively injected with primary human acute myeloid leukemia (AML) blasts and cord blood-derived human progenitor cells from healthy donors. These mice were then treated with TEG001 and mock cells. Tumor burden and human cells engraftment were measured in peripheral blood and followed up over time by quantifying for absolute cell number by flow cytometry. Statistical analysis was performed using non-parametric 2-tailed Mann-Whitney t-test.

Results: We successfully engrafted primary AML blasts and healthy hematopoietic cells after 6-8 weeks. Here we report that metabolic cancer targeting through TEG001 eradicated established primary leukemic blasts in vivo, while healthy hematopoietic compartments derived from human cord-blood remained unharmed in spite of TEGs persistence up to 50 days after infusion. No additional signs of off-target toxicity were observed in any other tissues. Conclusion: Within the limitations of humanized PD-X models, targeting CD277J by TEG001 is safe and efficient. Therefore, we have initiated clinical testing of TEG001 in a phase I first-in-human clinical trial (NTR6541; date of registration 25 July 2017).

Keywords: AML, Immunotherapy, TEGs, Mice model, Preclinical, Toxicity, TCR engineering

\footnotetext{
* Correspondence: j.h.e.kuball@umcutrecht.nl

†Inez Johanna and Trudy Straetemans are contributed equally to this work.

'Zsolt Sebestyen and Jürgen Kuball are both senior authors.

'Department of Hematology and Laboratory of Translational Immunology,

University Medical Center Utrecht, Utrecht, The Netherlands

Full list of author information is available at the end of the article
}

(c) The Author(s). 2019 Open Access This article is distributed under the terms of the Creative Commons Attribution 4.0 International License (http://creativecommons.org/licenses/by/4.0/), which permits unrestricted use, distribution, and reproduction in any medium, provided you give appropriate credit to the original author(s) and the source, provide a link to the Creative Commons license, and indicate if changes were made. The Creative Commons Public Domain Dedication waiver (http://creativecommons.org/publicdomain/zero/1.0/) applies to the data made available in this article, unless otherwise stated. 


\section{Background}

Adoptive cell therapy with engineered immune cells targeting hematological malignancies entered clinical practice [1]. Reprogramming immune cells has been achieved so far with chimeric antigen-reactive receptors [2] and tumor-specific $\alpha \beta T$ cell receptors (TCRs) [3, 4]. However, the CAR-T concept frequently targets ubiquitously expressed antigens like CD19 for B cell malignancies [5], or FLT-3 [6] for acute myeloid leukemia (AML), as well as stress antigens like NKG2D (natural-killer group 2, member D) for a broader range of cancers [7], raising the question of whether such strategies result as collateral damage in either the long-term deletion of essential hematopoietic subsets or within the context of physiological or therapeutic stress like irradiation to self-reactivity. Given the low mutational load of AML [8], the targeting of neo-antigens has not been successful, and targeting of minor antigens like HA-1 allows only the inclusion of a minority of patients [9]. Thus, novel strategies are needed to attack myeloid malignancies within the context of engineered immune cells.

One very attractive and so far, not well-explored alternative to mediate tumor-specific TCR derives from unconventional $\gamma 9 \delta 2 \mathrm{~T}$ cells subsets [10]. $\gamma 9 \delta 2 \mathrm{~T}$ cells sense molecular stress signatures via the accumulation of intracellular phosphoantigens level on infected and malignant cells [11]. This cell subset has the ability to kill tumor cells originating from hematological and solid malignancies in vitro, making it a promising immunotherapeutic option $[10,12]$. While several clinical trials have been conducted using ex vivo expanded and adoptively transferred autologous $\gamma 9 \delta 2 \mathrm{~T}$ cells in patients with advanced malignancies including AML, the results showed scarce activity [13]. One major obstacle has been the limited proliferative capacity of $\gamma 9 \delta 2 \mathrm{~T}$ cells in advanced cancer patients [14], as well as the underestimation of the substantial molecular and functional diversity within this subset $[15,16]$. Therefore, alternative strategies are needed for the clinical translation of the strong antitumor reactivity of receptors expressed on $\gamma 9 \delta 2 \mathrm{~T}$ cells [15].

To override the major weakness of $\gamma 9 \delta 2 \mathrm{~T}$ cells for its defective proliferative capacity and underestimated diversity, our group demonstrated that $\alpha \beta \underline{T}$ cells engineered to express a defined $\chi \delta T C R$, so-called TEGs, solves the proliferation deficiency and diversity of $\gamma 9 \delta 2 \mathrm{~T}$ cells by utilizing one defined $\gamma 9 \delta 2$ TCR with strong antitumor reactivity and the strong proliferative capacity of $\alpha \beta T$ cells. Furthermore, by utilizing $\alpha \beta \mathrm{T}$ properties, we retain both $\mathrm{CD} 4^{+}$and $\mathrm{CD}^{+}$effector cell functions in our TEGs. The first clinical candidate of TEGs derived from clone 5 (TEG001) has been shown to mediate the highest antitumor reactivity against a broader panel of tumor cells in vitro and in cell line-derived xenograft mouse models and to outperform natural $\gamma 9 \delta 2 \mathrm{~T}$ cells $[10,12,17,18]$. However, the assessment of the true activity of TEG001 against primary leukemia as well as potential toxicity in physiologically more relevant models has not been assessed so far, but is essential prior to entering a first-in-human clinical trial. Low toxicity of natural $\gamma 9 \delta 2 \mathrm{~T}$ cells in many clinical trials [13] cannot be used as an argument for safety, given also their lack of activity in men, mainly orchestrated through many NK-like immune inhibitory receptors expressed at the cell surface of natural $\gamma 9 \delta 2 \mathrm{~T}$ cells [16]. The major driver of the activity of TEG001, but also its potential risk of toxicity, is derived from the concept of utilizing a highly active $\gamma 9 \delta 2 \mathrm{TCR}$ out of the context of the natural brakes of a $\gamma 9 \delta 2 \mathrm{~T}$ cells, which have been also the pitfalls for their successful clinical translation to date. Thus, the key obstacle of clinical translation of TEG001 remains the assessment of its bare activity against primary leukemia as well as potential side effects against e.g. healthy hematopoietic compartments. Classical concept of efficacy and safety testing fail for this novel type of tumor-specific antigen, given that a joint conformational and spatial change of CD277 (later referred to as CD277J) mediated through early metabolic changes in cancer cells is recognized by the utilized ү982TCR $[12,19,20]$ and no tools are available to directly assess CD277J. To date, only cellular re-localization of RhoB can serve as a surrogate marker of CD277J [12]. Antibodies used for detecting CD277 rather induce or inhibit the conformational and spatial changes of CD277J $[21,22]$, thus they do not have the intrinsic ability to sense these alterations. Soluble $\gamma \delta$ TCR have been suggested to sense CD277J [23], however a more comprehensive analysis of such tools could not confirm their suitability, most likely due to the low affinity of the $\gamma 9 \delta 2 T C R$ (J Kuball unpublished observation). To remove these obstacles before clinical testing, we developed models which allow us to assess efficacy and toxicity of TEG001 in more physiological relevant environments, with primary tumor tissues as well as primary healthy cells. One example is the recently established 3D bone marrow model which enabled us to determine the efficacy of TEG001 against primary multiple myeloma cells, and to simultaneously exclude toxicity against stroma and endothelial cells in the bone marrow niche [24]. However, limited information is available when assessing activity of TEG001 against established leukemia, and toxicity against the complete hematopoietic compartment. Therefore, we utilized in this report an in vivo patient-derived xenograft (PD-X), and a healthy donor-derived xenograft (HD-X) model for assessing the efficacy of TEG001 against primary leukemic blasts and toxicity against the complete hematopoietic compartment, to provide a rationale for first-in-human testing of TEG001.

\section{Materials \& methods}

\section{Functional $\mathrm{T}$ cell assay}

IFN $\gamma$ ELISPOT was performed using anti-human IFN $\gamma$ mAb1-D1K(I) and mAb7-B6-1 (II) (Mabtech) in accordance 
with the manufacturer's protocol. Effector and target cells (E:T 1:3) were incubated for $24 \mathrm{~h}$ with or without pamidronate (10 or $100 \mu \mathrm{M}$, Calbiochem) as indicated. Pamidronate was added in all our in vitro experiment in order to enhance TEGs activation as previously reported [10].

\section{RhoB distribution analysis using confocal microscopy}

Human $\mathrm{CD}_{34}{ }^{+}$progenitor cells from a healthy donor were subjected to different conditions as follows: 1 ) untreated; 2) overnight stimulation with $50 \mathrm{IU} / \mathrm{ml} \mathrm{IL-2}$ or 3) 1000 $\mathrm{IU} / \mathrm{ml}$ IFN $\gamma$; 4) overnight incubation in the presence of 5 mM Cyclophosphamide (Cy, Sigma-Aldrich Chemie NV, South Holland), or 5) $20 \mu \mathrm{M}$ Fludarabine-phosphate (Flu, Sigma-Aldrich Chemie NV, South Holland), or 6) Cy/Flu combination. Primary AML, B cells, T cells, and monocytes were exposed to $100 \mu \mathrm{M}$ pamidronate and all cells were subsequently loaded to poly-L-lysine-coated coverslips. Attached cells were fixed, permeabilized and stained with a rabbit polyclonal anti-RhoB antibody (AbCam) followed by a secondary Goat anti-Rabbit IgG AlexaFluor488-conjugated antibody (Jackson ImmunoResearch). Cells were also stained with DAPI for nuclear staining. Intracellular RhoB distribution was visualized by confocal microscopy. RhoB signal ratios between intra-nuclear and extra-nuclear compartments were quantified using ImageJ software as described previously [12].

\section{Animal models}

NOD.Cg-PrkdcscidIl2rgtm1Wjl/SzJ (NSG) and NOD.CgPrkdcscidIl2rgtm1WjlTg(CMVIL3,CSF2,KITLG) 1Eav/Ml oySzJ (NSG-SGM3) mice originally obtained from Jackson Laboratory (Bar Harbor, ME, USA) were bred and housed in the specific pathogen-free (SPF) breeding unit of the Central Animal Facility of Utrecht University. Experiments were conducted according to Institutional Guidelines under acquired permission from the local Ethical Committee and per current Dutch laws on Animal Experimentation. Mice were housed in sterile conditions using an individually ventilated cage (IVC) system and fed with sterile food and water. Irradiated mice were given sterile water with antibiotic ciproxin for the duration of the experiment. Mice were randomized with equal distribution by sex and divided into 5 mice/group (for efficacy study) or 10 mice/group (for safety study).

Adult mice (10-14 weeks old) received sublethal total body irradiation (1.75 Gy) on Day 0. On Day 1, NSG mice were injected intravenously with $5 \times 10^{6} \mathrm{CD} 3$-depleted primary AML blast from donor p25 (efficacy study as PD-X model) or $0.25 \times 10^{6}$ healthy human $\mathrm{CD}_{4} 4^{+}$cells from six different donors (safety study as HD-X model). Engraftment and tumor burden were followed up in the peripheral blood as described in the subsection below. When the arbitrary threshold of 500 cells $/ \mathrm{ml}$ was reached, treatment was initiated. Mice received 2 injections of $10^{7}$ therapeutic TEG001 cells or TEG-LM1 mock cells (non-functional $\gamma \delta$ TCR-transduced $\mathrm{T}$ cells that carries length mutation of on the complementary determining region 3 (CDR3) region of the $\delta 2$-chain [18]). For second PD-X model for efficacy study, adult NSG-SGM3 mice received 2 injections of $10^{7}$ therapeutic TEG001 cells or TEG-LM1 mock cells at Day 8 and 16. All mice received $0.6 \times 10^{6} \mathrm{IU}$ of IL-2 (Proleukin; Novartis) in IFA subcutaneously together with the first $\mathrm{T}$ cell injection and every 21 days until the end of the experiment. Pamidronate $(10 \mathrm{mg} / \mathrm{kg}$ body weight) was injected intravenously together with the first $\mathrm{T}$ cell injection, and every 21 days until the end of the experiment. Pamidronate was added in all our in vivo experiment in order to enhance TEGs activation as previously reported [10]. Mice were routinely monitored at least twice a week for weight loss and symptoms of disease (sign of paralysis, weakness, and reduced motility).

\section{Cytology staining and analysis}

Cytopathologic evaluation of mouse bone marrow cytospin was performed by May-Grünwald Giemsa staining. Each sample was qualitatively and semi-quantitatively evaluated based on the following criteria: 1) cellularity ( 1 =high; 2 = moderate; $3=$ low); 2 ) presence of megakaryocytes; 3 ) presence of all cell lineages; 4) presence of all stages of maturation for each cell lineage; 5) description of the cell types present for each cell lineage.

\section{Histology staining and analysis}

Histopathologic evaluation was performed by hematoxylin and eosin (H\&E) staining for the following mouse tissues: liver, spleen, small (duodenum, jejunum, ileum) intestine. Each organ was semi-quantitatively evaluated based on the following criteria: 1) histologic lesions were semi-quantitatively assessed (grade: $0=$ absent; $1=$ minimal; $2=$ mild; 3 =moderate; $4=$ marked); 2) the inflammation was evaluated considering the distribution (focal, multifocal, multifocal to coalescing, diffuse), severity (grade 1-4) and cell type (lymphocytes, plasma cells, macrophages, neutrophils); 3) the presence of leukemic cell infiltrate.

\section{Statistical analysis}

Data were analyzed using GraphPad Prism (GraphPad Software Inc.) and represented as mean \pm standard deviation (SD) or standard error of mean (SEM) with * $P<0.05$; ** $P<0.01$; and $\% P<0.001$. Differences between groups were assessed using a two-way ANOVA, non-parametric 2-tailed Mann-Whitney t-test or Kruskal-Wallis test and Dunn's multiple comparison test where indicated.

Cell lines, primary human materials, retroviral transduction and depletion of non-engineered $\mathrm{T}$ cells, CFU assays, flow cytometry analysis, assessment for human cell engraftment and preparation of single cell suspensions are described in Additional file 1. 


\section{Results}

In vitro and in vivo activity of TEG001 against primary AML

Approximately $50 \%$ of the primary AML blasts tested so far are susceptible to TEG001 ( [17] and unpublished observation). We first confirmed activity of TEG001 against the primary AML blasts from multiple donors (Additional file 2: Table S1) by performing an IFN $\gamma$ ELIspot assay in the presence or absence of $10 \mu \mathrm{M}$ pamidronate (PAM) while the negative control (healthy $\mathrm{T}$ cells) was not recognized. Aminobiphosphonate compounds, including clinically used pamidronate, further accumulate intracellular phosphoantigens level [11]. Based on our previous study [10], the application of therapeutic concentrations of PAM enhances $\gamma 9 \delta 2 \mathrm{TCR}$ recognition, including TEG001. Daudi served as a positive control. Most of the primary AML blasts could induce significant IFN $\gamma$ production by TEG001 in the presence of PAM (Fig. 1A). Furthermore, we tested the cytolytic activity of TEG001 against primary AML blasts from donor p2. Primary AML blasts were incubated with either bulk $\alpha \beta$ T cells (as mock control) or with TEG001 cells in the presence of PAM on the methylcellulose matrix for the colony formation assay. Colonies were counted 8 days later. TEG001 showed a superior reduction of AML blast as shown by less colony formation in comparison to mock T cells (Fig. 1B). This result aligns with our previous data in which $\gamma 9 \delta 2 \mathrm{TCR}$-transduced $\alpha \beta \mathrm{T}$ cells inhibited colony forming unit (CFU) of primary AML blast [10].

From this screening, we selected AML blasts from patient 25 (p25) because of its initial susceptibility to TEG001, as well as its availability in sufficient numbers for further testing in mice. Next, we injected CD3-depleted primary AML blasts from p25 into irradiated mice intravenously (Fig. 1C). Engraftment and leukemia outgrowth were detected by measuring specific AML markers huCD $45^{+} \mathrm{CD} 13^{+} \mathrm{CD} 33^{+}$in peripheral blood by flow cytometry (Additional file 3: Figure S2). When 500 AML cells $/ \mathrm{ml}$ were detected in peripheral blood, treatment was initiated. Mice received two injections of TEG001 or TEG-LM1 mock in the presence of PAM and IL-2 (for the first TEGs injection) to support TEGs activation and proliferation in vivo. TEG-LM1 carries $\gamma 9 \delta 2 T C R$ with length mutation of on the CDR3 of the $\delta 2$-chain, which abrogates its function [18] and therefore chosen as a suitable mock control. $\gamma \delta \mathrm{TCR}$ expression for both TEG001 and TEG-LM1 mock is comparable, which subsequently infused into the mice (Additional file 4: Figure S1). In the peripheral blood of the TEG001-treated mice, primary AML cells were no longer detectable five weeks after TEGs infusion, but remained measurable in mock-treated mice (Fig. 1D), suggesting that in the described PD-X model TEG001 specifically eliminates primary AML blasts over time. We further addressed the influence of microenvironment to TEG001 recognition against primary AML blasts and developed a separate PD-X model using the same p25 AML in NSG-SGM3 mice that express human cytokines (i.e. IL-3, granulocyte/ macrophage colony-stimulating factor (GM-CSF), and stem cell factor (SCF)) that support better engraftment of AML blast in vivo [25]. Similarly, mice received two injections of TEG001 or TEG-LM1 mock in the presence of PAM and IL-2 (for the first TEGs injection) at Day 8 and Day 16 (Additional file 5: Figure S3A). While we did not see elimination primary AML blasts over time, TEG001-treated mice consistently showed lower AML burden in comparison to mock-treated mice as measured in peripheral blood (Additional file 5: Figure S3B). This result demonstrates antitumor activity of TEG001 against primary AML blasts in vivo as shown in two independent PD-X models.

\section{Assessing the activity of TEG001 against healthy hematopoiesis in vitro}

Next, we aimed to assess the toxicity of TEG001 against the hematopoietic compartment in vitro. Therefore, TEG001 and mock transduced T cells were incubated with the physiological hematopoietic target of $\gamma \delta$ T cells, namely $\mathrm{CD}_{14}{ }^{+}$monocytes, activated $\mathrm{T}$ cells as well as non-activated and activated $B$ cells in the absence and presence of PAM. Similar to the efficacy study, we include the presence of PAM to enhance TEG001 recognition as previously shown [10]. Daudi served again as a positive control. In an IFNy ELIspot assay cytokine secretion was only observed against the positive control and $\mathrm{CD} 14^{+}$ monocytes in the presence of PAM, while other T and B cells did not induce IFN $\gamma$ production (Fig. 2A).

Translocation of RhoB towards the cell membrane has been described as a key step for the recognition of a potential target by a $\gamma 9 \delta 2 \mathrm{TCR}$ [12]. This insight allowed us to test whether an additional stress of hematopoietic cells would activate this key step in the mode of action and thereby facilitate recognition of healthy compartments. As "stress" we have chosen irradiation, which is well known to activate many innate danger signals like MHC-like molecules [26], and is frequently used as preconditioning before the transfer of immune cells [27]. Therefore, we assessed the translocation of RhoB towards the cell membrane in T cells, B cells and CD14 ${ }^{+}$ monocytes in the absence and presence of irradiation. No significant increase in translocation of RhoB to the cell membrane could be observed (Fig. 2B) for the tested healthy hematopoietic cells. We also assessed the RhoB localization in healthy $\mathrm{CD}^{+} 4^{+}$progenitor cells upon stimulation with cytokines, such as IL-2 and IFN $\gamma$, as well as the presence of chemotherapy agents $\mathrm{Cy} / \mathrm{Flu}$ and compared to primary AML blast from donor p25. While 


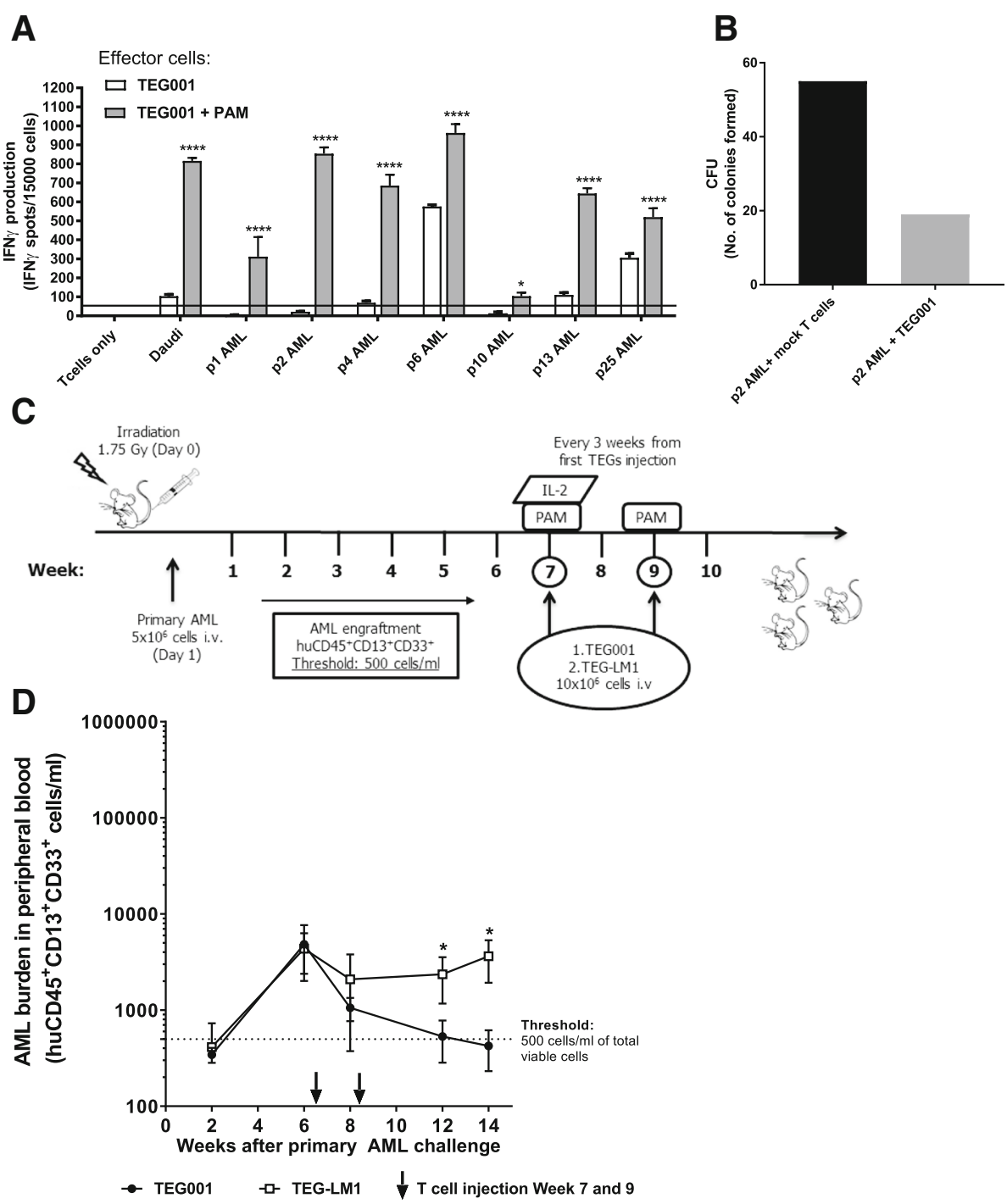

Fig. 1 In vitro and in vivo efficacy profile of TEG001. (a) Antitumor reactivity of TEG001 towards patient-derived primary AML blasts in vitro. Effector cells TEG001 and primary AML blasts from multiple donors (E:T ratio is 1:3) were incubated for $24 \mathrm{~h}$ with or without $10 \mu \mathrm{M}$ PAM as indicated. Daudi and healthy $T$ cells were included as positive and negative target controls, respectively. IFNy secretion was measured by ELISPOT. IFNY spots per 15,000 T cells are shown as mean \pm SD of at least 3 independent replicates for each target. Fifty spots/15,000 cells were considered as a positive antitumor response and indicated by the black horizontal line. Statistical significances were calculated by two-way ANOVA; ${ }^{*}, P<0.05 ;{ }^{* *}, P<0.01 ;{ }^{* * *}$, $P<0.001$; ${ }^{* * *}, P<0.0001$; (b) Bulk a TT cells (as mock T cells) or TEG001 cells were incubated with primary AML blast from donor p2 for $5 \mathrm{~h}$ at E:T ratio 10:1. Then cells were plated in methylcellulose-based medium and, after 8 days, colony formation was quantified using an inverted microscope. Shown is the number of CFU formed. Data is the result of a single experiment from single primary AML donor; (c) Schematic overview of in vivo experiment. NSG mice were irradiated at day 0 and engrafted with primary AML cells at day 1. AML cells were followed-up in the peripheral blood by flow cytometry. When the average AML cells were $>500 \mathrm{cell} s / \mathrm{ml}$, treatment was initiated. Mice received 2 injections of therapeutic TEG001 or TEG-LM1 mock in the presence of PAM (at week 7 and 9) and IL-2 (at week 7); (d) Tumor burden for primary AML was measured in peripheral blood by quantifying for absolute cell number by flow cytometry. Data represent mean \pm SD of all mice per group $(n=5)$. Statistical significances were calculated by non-parametric 2-tailed Mann-Whitney t-test; ${ }^{*}, P<0.05 ;{ }^{* *}, P<0.01 ;{ }^{* *}, P<0.001 ;{ }^{* * * *}, P<0.0001$

there is a significant increase in RhoB localization towards cell membrane in p25 AML blast, there are no significant increased for the healthy $\mathrm{CD} 34^{+}$progenitor cells in all conditions (Fig. 2C). Furthermore, the recognition profile by TEG001 of the same cell subsets after irradiation was assessed by cytokine secretion. Recognition of a priori non-recognized cells was not induced and recognition of $\mathrm{CD}_{1} 4^{+}$monocytes was slightly reduced after irradiation (Fig. 2D). Overall, our results suggest that TEG001 does not attack subsets of healthy hematopoiesis in the absence or presence of stress. Only $\mathrm{CD} 14^{+}$monocytes can be recognized in the presence of PAM as reported also for natural $\gamma 9 \delta 2 \mathrm{~T}$ cells [10]. 

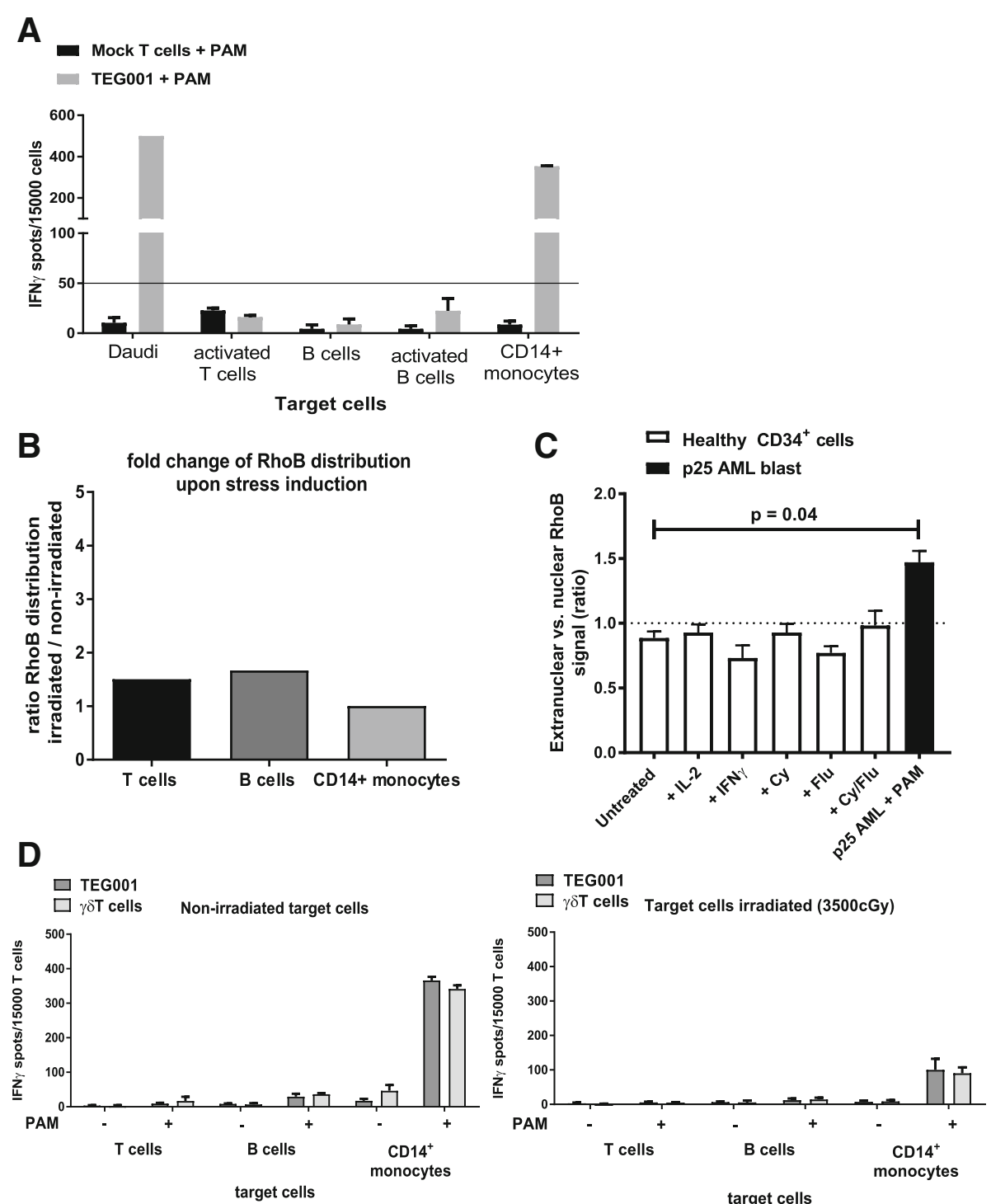

Fig. $\mathbf{2}$ In vitro safety profile of TEG001. (a) Comparable recognition profile of Daudi tumor cells versus healthy hematopoietic cells. Effector cells TEG001 and target cells (E:T ratio 1:3) were incubated for $24 \mathrm{~h}$ in the presence of $10 \mu \mathrm{M}$ PAM. IFNY secretion was measured by ELISPOT. IFNY spots per 15,000 T cells are shown as mean \pm SD of at least 3 independent replicates for each target. Fifty spots/15,000 cells were considered as a positive recognition response and indicated by the black horizontal line. (b) RhoB distribution for healthy hematopoietic cells upon irradiation as analyzed by confocal microscopy in the presence of $10 \mu \mathrm{M}$ PAM. Data is shown as fold-changed of RhoB distribution between irradiated cells (cellular stress condition) compared to non-irradiated cells from average ratio of at least ten different cells; (c) $\mathrm{CD}_{3} 4^{+}$progenitor cells from a

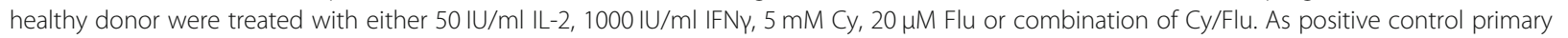
AML blast from donor p25 was treated with PAM. All cells were analyzed for intracellular distribution of RhoB using confocal microscopy. White bars represent healthy $\mathrm{CD} 34^{+}$progenitor cells, while black bar indicate primary AML blast (p25 AML). The RhoB signal ratio between nuclear and extranuclear cellular compartments was measured using ImageJ image analysis software. Graphs show average ratios of at least ten different cells \pm SEM. Statistical significance compared to untreated CD34 ${ }^{+}$progenitor cells was determined by using Kruskal-Wallis test and Dunn's multiple comparison test; (d) Comparable recognition profile of healthy hematopoietic cells in non-stressed (left panel) and stressed (irradiated, right panel) conditions. Effector cells TEG001 and target cells (E:T ratio 1:3) were incubated for $24 \mathrm{~h}$ in the presence of $100 \mu \mathrm{M}$ PAM. IFNY secretion was measured by ELISPOT. IFNy spots per 15,000 T cells are shown as mean \pm SD of at least 3 independent replicates for each target

\section{In vivo pharmacology and toxicology of TEG001}

Assessment of different hematopoietic subsets by TEGs in vitro is very restricted due to the limited sub-fractions available for testing. In addition, it does not allow for assessment of whether early precursors are affected. Therefore, we established a HD-X model with human cord-blood derived $\mathrm{CD} 4^{+}$progenitor cells from six healthy donors repopulated in irradiated mice to further assess the safety profile of TEG001 against the hematopoietic compartment (Fig. 3A). Engraftment of human leukocytes $\left(\right.$ huCD $45^{+}$) and other 

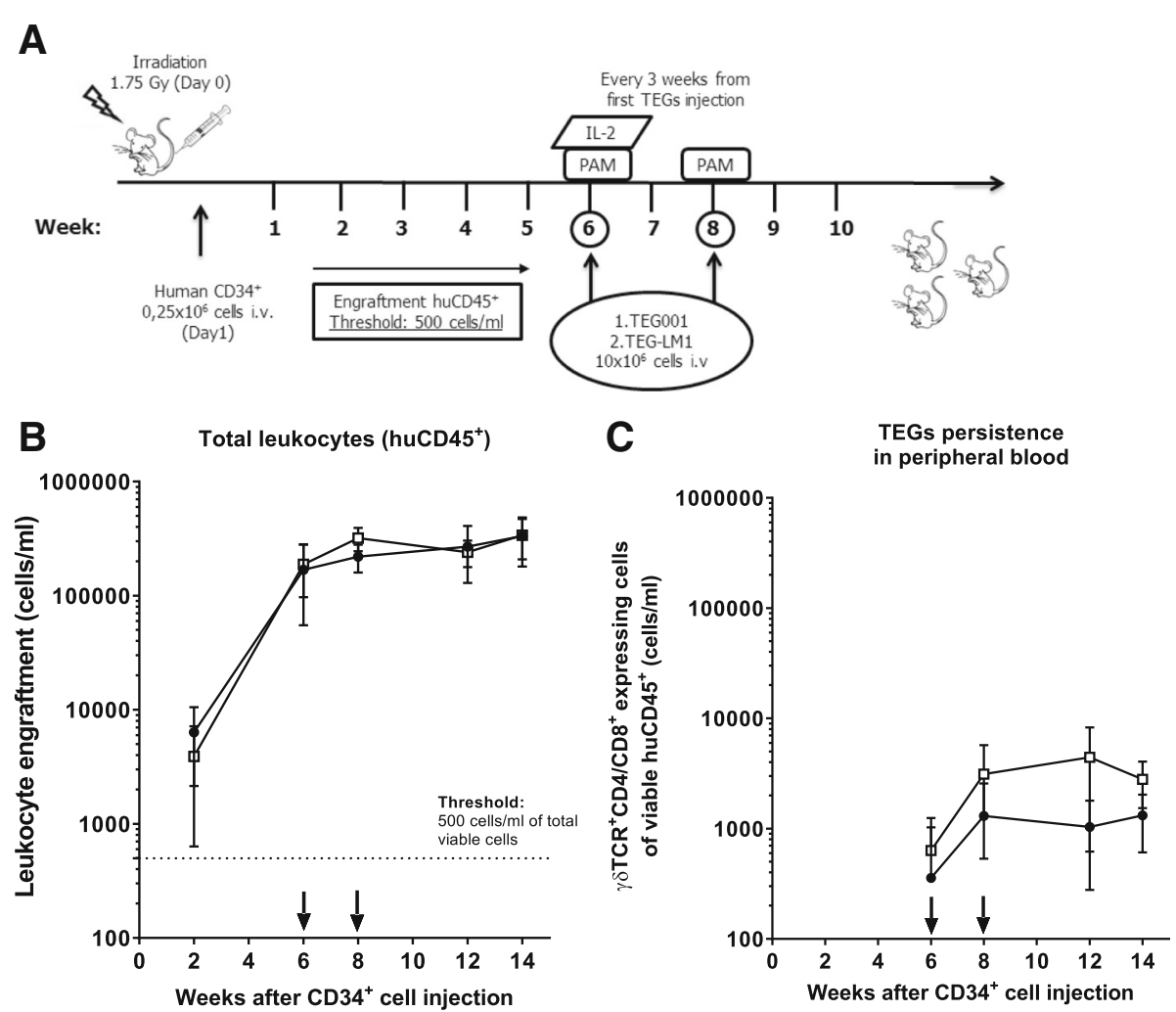

TEG001

$\rightarrow$ TEG-LM1

T cell injection Week 6 and 8

Fig. 3 In vivo safety profile of TEG001. (a) Schematic overview of the safety experiment in healthy donor-derived xenograft (H-DX) model. NSG mice were irradiated at day 0 and engrafted with healthy cord blood-derived CD34 ${ }^{+}$progenitor cells on day 1 . Engraftment was followed-up in peripheral blood by flow cytometry and when $>500$ huCD $45^{+}$cells $/ \mathrm{ml}$ were present, mice received 2 injections of therapeutic TEG001 or TEGLM1 mock in the presence of PAM (at week 6 and 8) and IL-2 (at week 6); (b-c) In vivo safety profile of TEG001 towards healthy human hematopoietic cells. Healthy human cells engrafted in NSG mice (b) with long-term persistence of TEGs in peripheral blood (c). Data represent mean \pm SD of all mice per group $(n=10)$

hematopoietic cellular subsets in peripheral blood was measured by flow cytometry (Additional file 6: Figure S4A and S4B). When 500 huCD $45^{+}$cells $/ \mathrm{ml}$ were detected in peripheral blood, treatment was initiated with either TEG001 or TEG-LM1 mock in the presence of PAM and IL-2 (for the first TEGs injection) to support TEGs activation and proliferation in vivo. While we observed a reduction of tumor burden by TEG001 (Fig. 1D), no significant differences in engraftment of healthy hematopoietic cells between treatment groups were observed up to 50 days after infusion when assessed by huCD $45^{+}$ (Fig. 3B). TEG001 as well as TEG-LM1 cells could be detected after injection until the end of the study period in the peripheral blood of mice (Fig. 3C).

Next, we investigated the reconstitution of diverse hematopoietic cellular subsets in vivo in more detail in the peripheral blood of mice. In particular, we were interested in the impact on monocytes given that in vitro natural $\gamma 9 \delta 2 \mathrm{~T}$ cells as well as TEG001 can recognize primary monocytes. Interestingly, neither $\mathrm{CD} 14^{+}$monocytes, nor
$\mathrm{CD} 9^{+} \mathrm{B}$ cells, $\mathrm{CD}^{+} \mathrm{T}$ cells, or $\mathrm{CD} 34^{+}$progenitor cells were impaired in outgrowth when comparing mice injected with TEG001 or TEG-LM1 (Fig. 4A-D). At the end of study period, we also obtained single cell suspension from spleen and bone marrow from three mice for both the TEG001 and TEG-LM1 mock group to analyze the reconstitution of similar cell subsets in more detail in primary tissues (Fig. 5A-E). In line with our observations in peripheral blood, we could observe all relevant subsets, namely $\mathrm{CD} 14^{+}$monocytes, $\mathrm{CD} 19^{+} \mathrm{B}$ cells, $\mathrm{CD}^{+} \mathrm{T}$ cells, and $\mathrm{CD} 34^{+}$progenitor cells.

We next collected bone marrow cytospin samples from the same mice for a more detailed cytopathology analyses. All of the bone marrow samples from both treatment groups showed a pleomorphic population of cells derived from erythroid and myeloid lineages, with all the maturation stages (Fig. 6A). In almost all samples (5/6) eosinophilic differentiation was also evident. Beside normal blasts, an immature population with altered morphology (dysplastic immune cells), consistent with granular blasts 

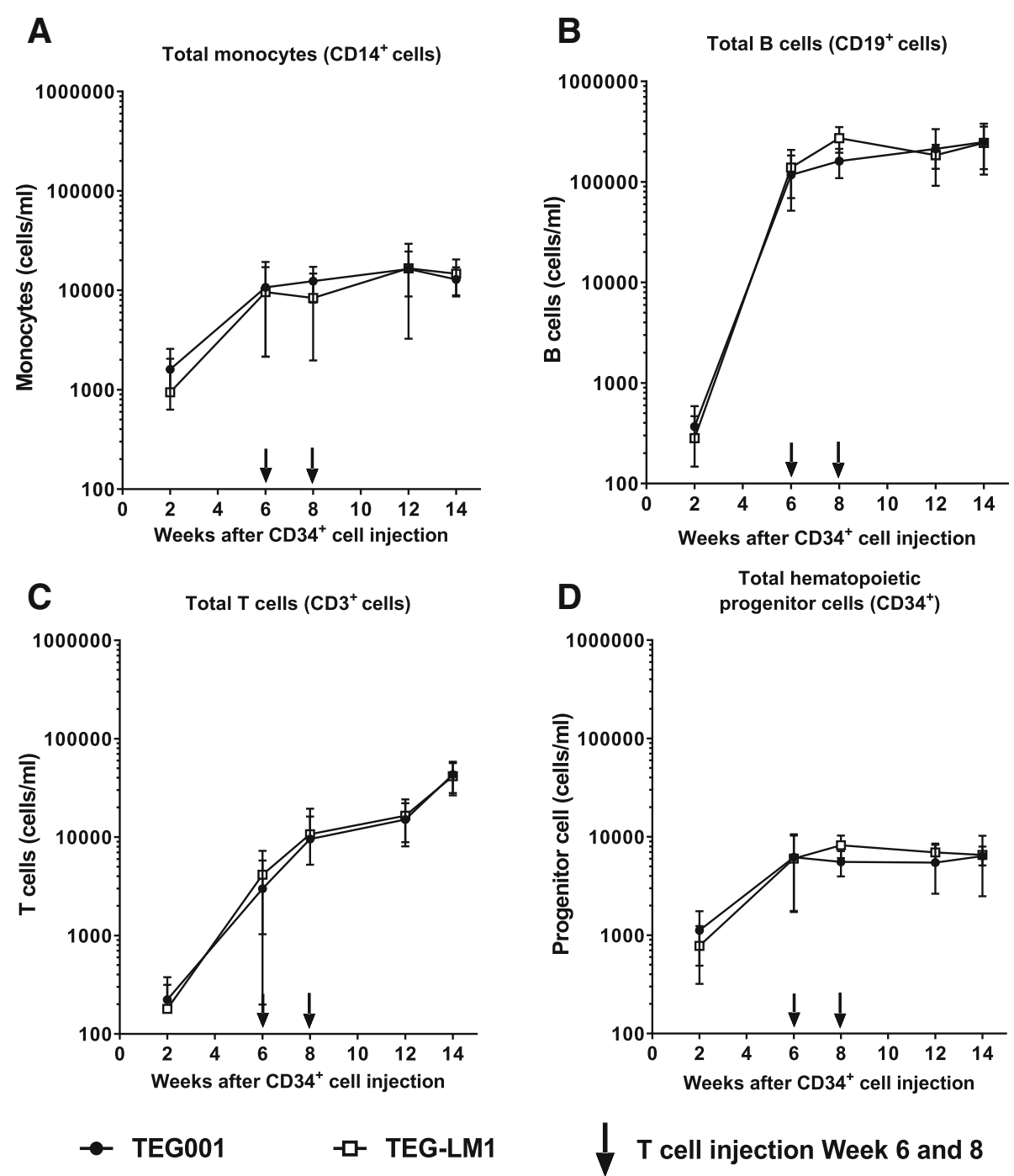

Fig. 4 In vivo reconstitution of healthy human hematopoietic compartments in peripheral blood. Comparable profile between TEG001 and TEGLM1 mock group of reconstituted healthy human hematopoietic cellular subsets, including CD14 ${ }^{+}$monocytes (a) B cells (b), T cells (c), and CD34 ${ }^{+}$ progenitor cells $(\mathbf{d})$ as measured by flow cytometry. Data represent mean \pm SD of all mice per group $(n=10)$

was detected in both TEG001 and TEG-LM1 treated mice as well as cells with blast-like phenotypes with an indented nucleous, consistent with promonocytes $(4 / 6)$, but no leukemic features were observed. Importantly, we did not observe any apparent differences in their outgrowth between treatment groups indicating that TEG001 do not harm the reconstitution of healthy hematopoietic compartments in vivo.

To evaluate off-target toxicity of TEG001 towards healthy tissues not related to the known mode of action which is absent in mice [21], we collected further mouse spleen, liver and intestine from three mice for each TEG001 and TEG-LM1 mock group and performed histopathology analyses. Spleen tissues showed non-neoplastic, lympho/histiocytic proliferative lesions in all the examined samples, of both treatment groups (Fig. 6B). Similarly, no significant histological features of toxicity or other relevant abnormalities were observed in liver or intestine in all samples (Fig. 6C-D). Most of the samples showed extramedullary hematopoiesis, mainly involving the erythroid lineage (extramedullary erythropoiesis) with scattered megakaryocytes sometimes evident, as a possible consequence of the engrafted human $\mathrm{CD} 34^{+}$progenitor cells in this mouse model. Overall, our data indicate there are no significant differences in histology features and notably, there are no off-target toxicities observed in all healthy tissues upon TEG001 treatment.

\section{Discussion}

TEG001 has been selected as the first candidate for clinical testing (NTR6541) based on its superior recognition of hematological malignancies against both cell lines and 

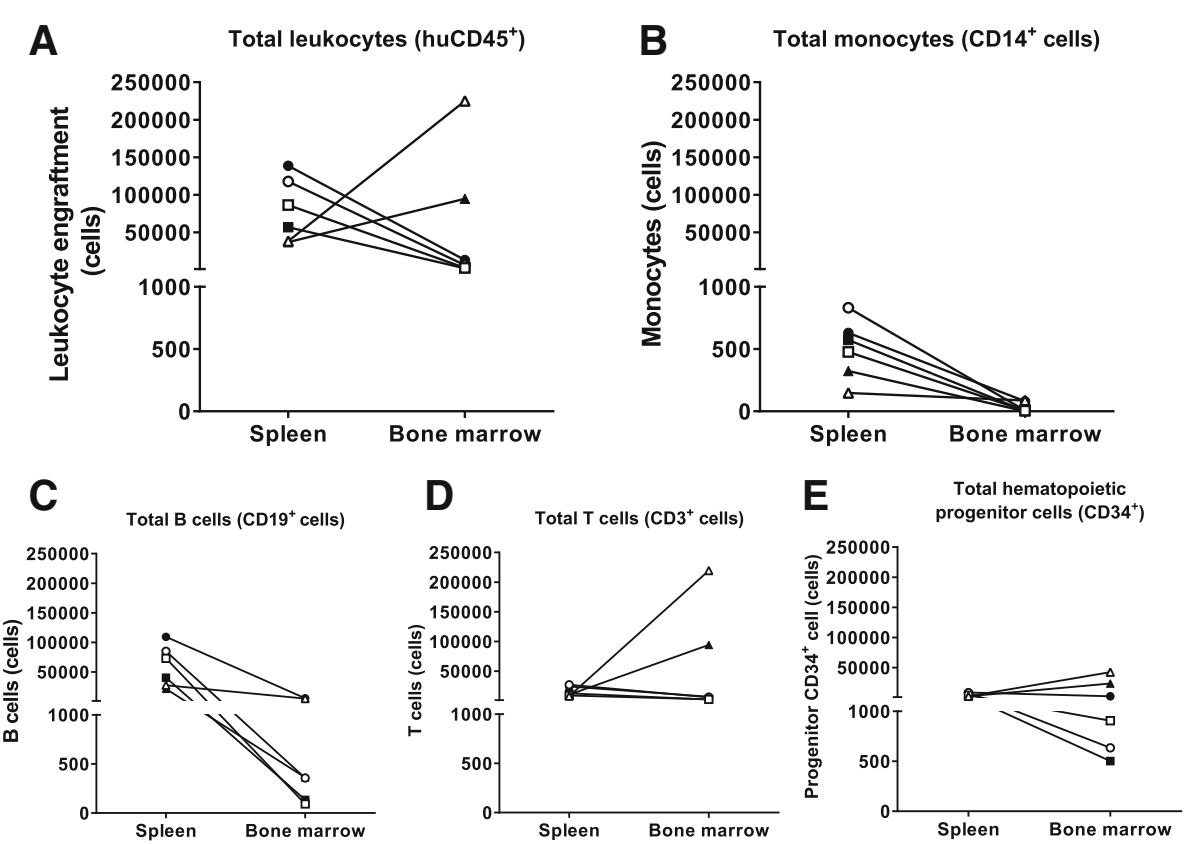

Fig. 5 In vivo reconstitution of healthy human hematopoietic compartments in tissues. Comparable profile between TEG001 and TEG-LM1 mock group of reconstituted healthy human hematopoietic cellular subsets in spleen and bone marrow, including total human CD45 ${ }^{+}$leukocytes (a), CD14 ${ }^{+}$monocytes (b), B cells (c), T cells (d) and CD34 $4^{+}$progenitor cells (e) as measured by flow cytometry. Shown in the data from individual mouse (represented by different symbols) of both TEG001 (filled symbol) and TEG-LM1 mock (open symbol) group ( $n=3$ mice/group)

primary AML in vitro and its ability to limit the tumor outgrowth in cell line-derived xenograft mouse models $[10,17,18]$. Within this study, we have been able, for the very first time, to assess therapeutic efficacy towards primary AML blasts in a clinically relevant model with established leukemic load in vivo, while excluding toxicity against other hematopoietic stem cell compartments. Our current observation that primary AML can be eliminated in an in vivo model by TEG001, without affecting the hematopoietic compartment, is in line with our previous observation that an alteration in the RhoB-CD277J axis, the putative ligand of $\gamma 9 \delta 2 \mathrm{TCR}$, is selectively observed in the leukemic but not healthy hematopoietic stem cell [12].

A major challenge a priori clinical testing of novel cell-based and gene therapy products remains to assess efficacy and toxicity in relevant pre-clinical models in order to avoid unwanted toxicities like those reported for different CAR-T [28] or $\alpha \beta T C R$ gene therapy programs [29]. This reflects the quite different characteristics of cell-based gene therapy medicinal products in comparison to conventional synthetic drugs. Thus, classical clinical considerations of therapeutic efficacy and safety assessments might no longer apply for these 'living' medicinal products. With TEG001, a next level of complexity is introduced due to the nature of the target. In contrast to, e.g., CD19-directed CAR T gene therapy, which targets a very well-defined protein expressed on cancer cells and $\mathrm{B}$ cells [5], TEG001 is targeting metabolic changes in stressed and malignant cells, driven by a dysregulated mevalonate pathway [11]. Although transfer of conventional $\gamma 9 \delta 2 \mathrm{~T}$ cells has not been reported to associate with substantial toxicity [13], the TEG concepts express an activating $\gamma 9 \delta 2 \mathrm{TCR}$ outside the context of its natural brakes, through a plethora of killer immunoglobulin-like receptor (KIR) inhibitory receptors usually operational in natural ү $9 \delta 2 \mathrm{~T}$ cells. Therefore, Dutch authorities have required additional safety tests for TEG001 prior to clinical testing. However, dysregulated metabolic pathways do not allow for high throughput evaluations of the ligand in all tissues through, e.g., gene expression or transcriptome analyses [30]. Consequently, following the advice of the Dutch authorities, our group developed different strategies to test the efficacy and safety of TEG001 in models where healthy and malignant cells are present either simultaneously or sequentially. One such model is a 3D bone marrow model where primary multiple myeloma cells grow out along with healthy stromal cells into an artificial bone marrow niche. Upon TEG001 injection, this model confirmed the activity of TEG001 against the malignant fraction, but not healthy bystander cells present in the bone marrow niche [24]. However, the 3D bone marrow niche is also limited, as it does not allow for engrafting of the complex hematopoietic system and or assessing toxicity against all cellular compartments usually generated from a hematopoietic stem cell. 


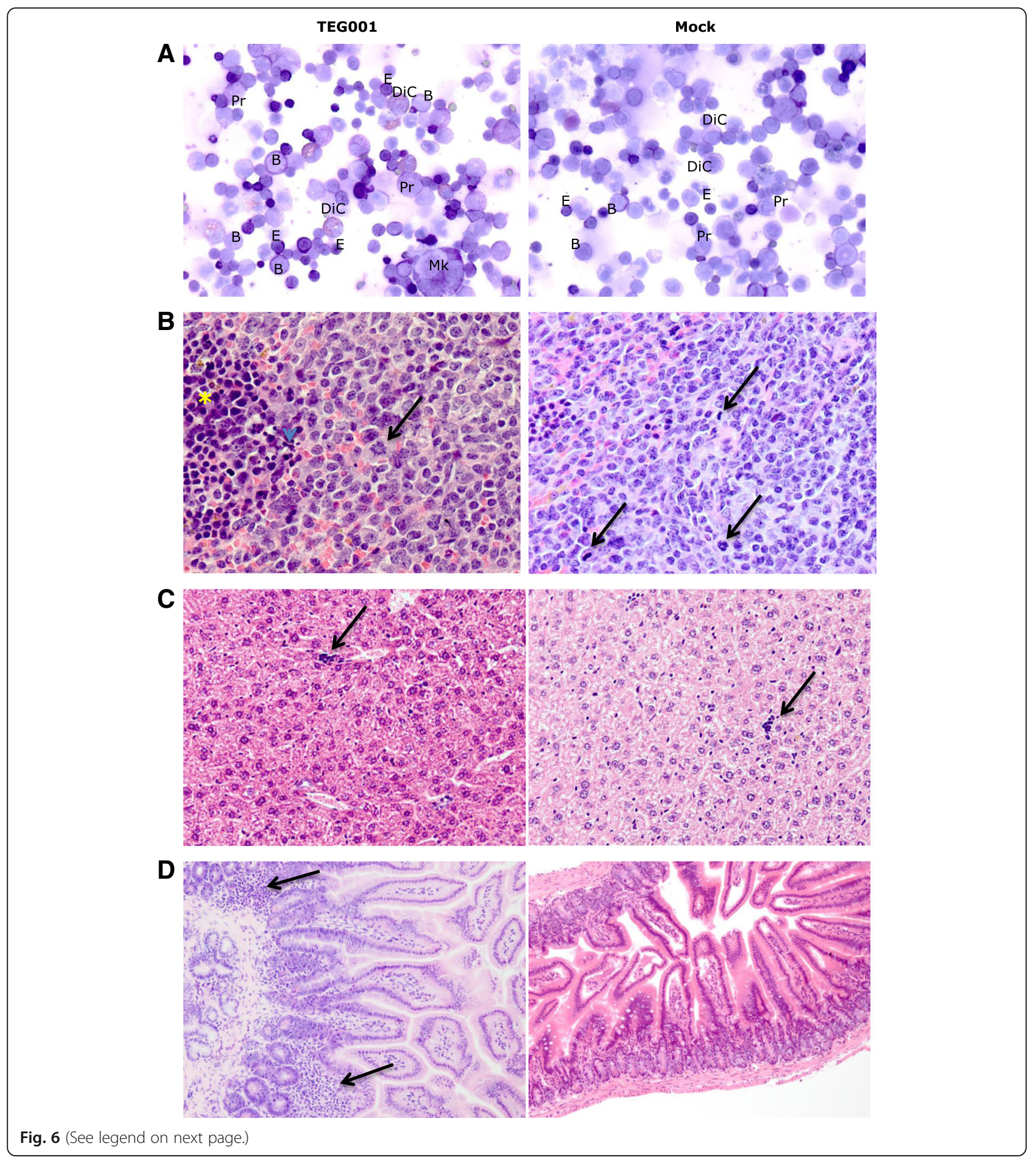


(See figure on previous page.)

Fig. 6 Cytopathology analysis of bone marrow and histopathology analysis of mouse vital organs (spleen, liver, intestine). (a) Representative picture of May-Grünwald Giemsa staining for bone marrow cytospin from both treatment groups (TEG001 and TEG-LM1 mock) with pleomorphic population of cells with all maturation stages including numerous blasts (B), promyelocytes (Pr), dysplastic immature cells (DiC), megakaryocytes (Mk) and a mixed population of myeloid and erythroid (E) lineages; (b) Representative pictures for H\&E staining of mouse spleen for both treatment group (TEG001 and TEG-LM1 mock) with non-neoplastic, lympho/histiocytic hyperplastic lesion with mitotic figure (arrows), apoptotic bodies (arrowhead) and erythroid precursors $\left(^{*}\right)$. Magnification: 40X; (c) Representative pictures for H\&E staining of mouse liver for both treatment group (TEG001 and TEG-LM1 mock) with small focus of extramedullary hematopoiesis (arrows) in all samples, which could be due to the mouse model with engraftment of human CD34 ${ }^{+}$progenitor cells. Magnification: 20X; (d) Representative pictures for H\&E staining of mouse intestine for TEG001-treated group (left) showing multifocal lymphocytic infiltration of lymphoid cells (arrows) in a small tract of the small intestine

(background lesion) and TEG-LM1 mock-treated group (right) with normal jejunum. Magnification: 10X. Shown are representative pictures from an individual mouse of both TEG001 and TEG-LM1 mock group ( $n=3$ mice/group) with no significant differences in overall cytopathology and histology features between treatment groups

To study the interaction between tumor and immune cells, we have to consider also their interaction within the same microenvironment. Xia and colleagues [31] develop humanized mice model with human hematopoietic system and autologous leukemia in the same individual mouse. This model is developed by transducing $\mathrm{CD} 34^{+}$ fetal liver cells with retroviral vector containing mixed-lineage leukemia MLL-AF9 fusion gene, which allows recapitulation of human leukemic diseases [31, 32]. Although it would be interesting to develop a similar humanized mouse model in which healthy human hematopoietic cells and primary leukemic blasts presence in the same individual mouse, the availability of healthy human $\mathrm{CD}_{34}{ }^{+}$progenitor cells from the very same leukemia patient is a limiting factor. Hence, we develop two separate mice models and thereby avoiding limiting criteria of HLA-matching between healthy CD $34^{+}$progenitor cells and primary AML donors.

In order to test the efficacy of TEG001, we utilized a mouse xenograft model, which has been widely used to study therapeutic responses in heterogeneous diseases such as cancer. PD-X models, considered to closely mimic human diseases, are established by engrafting primary patient material into immunodeficient mice [33]. Assessment of AML burden in mouse xenograft models is commonly performed by measuring the percentage of human leukemic cells in bone marrow at the end of study period. In this study, we developed a stringent treatment model where we infused TEG001 upon the onset of the disease (represented by an arbitrary threshold of 500 AML cells/ml detected in peripheral blood). Moreover, we developed an elegant method that allowed us to follow the disease progression for a longer period as well as the treatment effect to reduce tumor burden over time by measuring AML cells in peripheral blood. Nonetheless, we acknowledged some limitations in our method, such as variable engraftment rates commonly observed in PD-X model [34] and a low level of AML engraftment in peripheral blood of adult NSG mice as reported previously [35]. In spite of these limitations, we were able to detect a significant reduction of AML cells in peripheral blood of TEG001-treated mice in comparison to the mock-treated group. Furthermore, we developed a separate PD-X model using NSG-SGM3 mice using the same primary AML blast from donor p25 to assess the influence of microenvironment towards TEG001 efficacy profile. NSG-SGM3 mice express human cytokines, including IL-3, GM-SCF, and SCF, and thereby supporting primary AML engraftment and their survival in vivo [25]. Here we demonstrate that TEG001-treated group showed significantly lower AML burden in comparison to mock-treated group, despite the lack of tumor clearance. This could be due to the more protective microenvironment poses by NSG-SGM3 mice, which could hamper T cell access to target cells and therefore limit the ability of TEG001 to clear primary AML burden over time. Based on the overall data and thus as proof-of-principle we have demonstrated the efficacy profile of TEG001 against primary human AML in two independent models.

In order to assess the toxicity of TEG001 against the hematopoietic compartment in the very same model we engrafted NSG mice with CD34 ${ }^{+}$progenitor cells derived from healthy human cord blood donors. Reconstitution of hematopoietic cellular compartments when assessed in the peripheral blood occurred at different stages, in which $\mathrm{CD}_{14}{ }^{+}$monocytes and $\mathrm{CD}_{19^{+}} \mathrm{B}$ cells significantly increased two weeks after progenitor cell injection, whereas $\mathrm{CD}^{+} \mathrm{T}$ cells reconstituted relatively slower, however no differences could be observed between TEG001 and mock-treated mice. Furthermore, we investigated whether TEG001 does affect hematopoietic compartments in different tissues, specifically spleen and bone marrow, at which progenitor cells should reside [36, 37]. While we could find all equivalent cell subsets with comparable reconstitution for both treatment groups also in spleen and bone marrow, there were differences in the prevalence for CD $14^{+}$monocytes and $\mathrm{CD} 19^{+} \mathrm{B}$ cells in different tissues, however again with no difference between TEG001 and mock treated mice. Monocytes were found in higher numbers in the peripheral blood when compared to bone marrow and spleen, whereas B cells were prevalently observed in the periphery and spleen. This observation is in line with previous studies showing that the reconstitution of human hematopoietic 
stem cells in host mice is commences predominantly with erythroid and myeloid cells, followed by lymphoid progenitor and lastly mature lymphocytes [38]. Also, neither induction of cellular stress by irradiation nor exposure to inflammatory cytokines (i.e., IL-2 and IFN $\gamma$ ), or the presence of chemotherapy agent $\mathrm{Cy} / \mathrm{Flu}$ alter RhoB translocation towards the cell membrane for healthy $\mathrm{CD} 34^{+}$ progenitor cells, and thus no alteration of TEG001 recognition pattern. In addition, our data confirm that different tissue compartments are comprised of different types of immune cells; and show that TEG001 treatment did not influence this pattern. Thus TEG001 most likely does not affect homing of hematological subsets nor mediate hematopoietic toxicity, as suggested by our previous work demonstrating that the mode of action is mainly observed in tumor cells and not in the healthy hematopoietic compartment [12, 19]. The only physiological target of $\gamma 9 \delta 2 \mathrm{TCRs}$ are professional antigen presenting cells (APC) like monocytes and dendritic cells in the presence of PAM [18], as also demonstrated in this study in the in vitro experiments. However, as reported previously, this recognition apparently fosters the maturation of APC and potentially broadens an adaptive immune response through epitope spreading [39] rather than promoting elimination of APC. In line with this assumption, we could still detect $\mathrm{CD} 14^{+}$monocytes reconstitution in vivo after transfer of TEG001.

\section{Conclusion}

In conclusion, our data suggest antitumor reactivity of TEG001 against primary AML blasts in vivo. While we concur that the absence of evidence is not equal to the evidence of absence and within the limitation of our current models where off-target activities cannot be excluded entirely, there are no data indicating an increased safety risk specific for TEG001. A GMP-compliant production of TEG001 has now been established [17, 40], and will be used in an ongoing phase I open-label dose escalation study to explore toxicity and activity of TEG001 in patients with primary refractory or relapsed acute myeloid leukemia, as well as patients with multiple myeloma.

\section{Additional files}

Additional file 1: Supplementary Materials and methods including cell lines, primary materials, retroviral transduction and depletion of nonengineered T cells, CFU assay, flow cytometry analysis, assessment for human cell engraftment and Preparation of single cell suspensions. (DOCX $25 \mathrm{~kb}$ )

Additional file 2: Table S1. Primary AML characteristic. Characteristic of primary AML materials from different donors. (DOCX $17 \mathrm{~kb}$ )

Additional file 3: Figure S2. Gating strategy for flow cytometry analysis of primary AML burden. A representative flow cytometry plot of murine peripheral blood. Tumor load was measured by quantifying absolute cell number of viable huCD $45^{+} \mathrm{CD} 13^{+} \mathrm{CD} 33^{+}$of the primary $\mathrm{AML}$ blast and representative plot for TEG001 and TEG-LM1 mock group. (PPTX 178 kb)
Additional file 4: Figure S1. $\gamma \delta T C R$ expression of TEG001 and TEG-LM1 mock. A representative flow cytometry plot $\gamma \delta T C R$ expression of TEG001 and TEG-LM1 mock after transductions, after aßTCR depletion and prior to infusion into mice after 2 weeks expansion. (PPTX $191 \mathrm{~kb}$ )

Additional file 5: Figure S3. In vivo efficacy profile of TEG001 in PD-X model of primary blast in NSG-SGM3 mice. (A) Schematic overview of in vivo experiment. NSG-SGM3 mice were irradiated at day 0 and engrafted with primary AML cells at day 1. AML cells were followed-up in the peripheral blood by flow cytometry. Mice received 2 injections of therapeutic TEG001 or TEG-LM1 mock in the presence of PAM (at Day 8 and 16) and IL-2 (at Day 8); (B) Tumor burden for primary AML was measured in peripheral blood by quantifying for absolute cell number by flow cytometry. Data represent mean \pm SD of all mice per group ( $n=5$ mice/group). Statistical significances were calculated by non-parametric 2-tailed MannWhitney t-test; * $P<0.05$; **, $P<0.01$; ${ }^{* *}, P<0.001$; ****,

$P<0.0001$. (PPTX $101 \mathrm{~kb}$ )

Additional file 6: Figure S4. Gating strategy for flow cytometry analysis of healthy hematopoietic compartments. A representative flow cytometry plot of murine peripheral blood. (A) Engraftment was determined by quantifying absolute cell number of viable huCD45 ${ }^{+}$ of healthy stem cells; (B) Hematopoietic cellular compartments outgrowth were determined by quantifying absolute cell number for $\mathrm{CD}_{19}{ }^{+} \mathrm{B}$ cells, $\mathrm{CD}^{+} \mathrm{T}$ cells, and $\mathrm{CD} 14^{+}$monocytes. Also, persistence of TEGs were determined by quantifying absolute cell number for

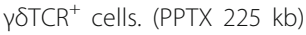

\section{Abbreviations}

AML: Acute myeloid leukemia; APC: Antigen presenting cells; CAR$\mathrm{T}$ : Chimeric antigen receptor $\mathrm{T}$ cells; CDR: Complementary Determining Region; CFU: Colony Forming Unit; Cy: Cyclophosphamide; FCS: Fetal calf serum; Flu: Fludarabine-phosphate; GMP: Good Manufacturing Practice; GM-SCF: Granulocyte/Macrophage Colony-Stimulating Factor;

H\&E: Hematoxylin and eosin; HD-X: Healthy donor-derived xenograft; IVC: Individually ventilated cage; KIR: Killer immunoglobulin-like receptor; NKG2D: Natural-killer group 2, member D; PAM: Pamidronate; PBMCs: Peripheral blood mononuclear cells; PD-X: Patient-derived xenograft; SCF: Stem Cell Factor; SD: Standard deviation; SEM: Standard error of mean; SPF: Specific pathogen-free; TCR: T cell receptor;

TEGs: aßT cells Engineered to express a defined $\gamma \delta T C R$

\section{Acknowledgements}

We thank M. Plantinga (UMC Utrecht, The Netherlands) for primary human cord-blood derived $\mathrm{CD}_{3} 4^{+}$materials and Halvard Boenig (Institute for Transfusion Medicine and Immunohematology, Goethe University, Frankfurt a. M., Germany) for providing feeder cells.

\section{Fundings}

Funding for this study was provided by ZonMW 43,400,003 and VIDI-ZonMW 917.11.337, UU 2013-6426, UU 2014-6790 and UU 2015-7601 and Gadeta to JK, and UU 2018-11,393 to ZS and JK.

\section{Availability of data and materials}

All data generated or analyzed during this study are included in this published article and its Additional files.

\section{Authors' contributions}

JK developed the conceptualization for the overall study. IJ and TS designed and developed the in vivo models. IJ, TS, SH performed the in vivo experiments. SH, TA, and ZS performed in vitro experiments. HN gave input and helped to setup for the in vivo model for primary $A M L$ (efficacy study). $L B$ and $A B$ performed the histopathology and cytopathology examination of the mouse tissues. IJ analyzed all in vivo data and was a major contributor in writing the manuscript. TS, ZS and JK supervised the study. IJ, TS, ZS, and JK wrote the manuscript; all authors read and approved the final manuscript.

\section{Ethics approval and consent to participate}

In vivo experiments were conducted according to Institutional Guidelines under acquired permission from the local Ethical Committee and per current Dutch laws on Animal Experimentation. 


\section{Consent for publication}

Not applicable.

\section{Competing interests}

JK received funding by Gadeta. JK and ZS are inventors on different patents with $\gamma \delta T C R$ sequences, recognition mechanism and isolation strategies. JK is CSO and shareholder of Gadeta.

\section{Publisher's Note}

Springer Nature remains neutral with regard to jurisdictional claims in published maps and institutional affiliations.

\section{Author details \\ 'Department of Hematology and Laboratory of Translational Immunology, University Medical Center Utrecht, Utrecht, The Netherlands. ${ }^{2}$ Faculdade de Medicina, Instituto de Medicina Molecular, Universidade de Lisboa, Lisbon, Portugal. ${ }^{3}$ Department of Pathobiology, Faculty of Veterinary Medicine, Dutch Molecular Pathology Center, Utrecht University, Utrecht, The Netherlands.}

\section{Received: 17 August 2018 Accepted: 4 March 2019}

\section{Published online: 12 March 2019}

\section{References}

1. Chabannon C, Kuball J, Bondanza A, Dazzi F, Pedrazzoli P, Toubert A, et al. Hematopoietic stem cell transplantation in its 60s: a platform for cellular therapies. Sci Transl Med. 2018;10(436).

2. Srivastava S, Riddell SR. Engineering CAR-T cells: design concepts. Trends Immunol. 2015;36(8):494-502.

3. Kuball J, Hauptrock B, Malina V, Antunes E, Voss RH, Wolfl M, et al. Increasing functional avidity of TCR-redirected T cells by removing defined $\mathrm{N}$ glycosylation sites in the TCR constant domain. J Exp Med. 2009;206(2):463-75.

4. Kuball J, Dossett ML, Wolfl M, Ho WY, Voss RH, Fowler C, et al. Facilitating matched pairing and expression of TCR chains introduced into human T cells. Blood. 2007;109(6):2331-8.

5. Turtle CJ, Maloney DG. Clinical trials of CD19-targeted CAR-modified T cell therapy; a complex and varied landscape. Expert Rev Hematol. 2016;9(8): $719-21$

6. Jetani H, Garcia-Cadenas I, Nerreter T, Thomas S, Rydzek J, Meijide JB, et al. CAR T-cells targeting FLT3 have potent activity against FLT3(-)ITD(+) AML and act synergistically with the FLT3-inhibitor crenolanib. Leukemia. 2018; 32(5):1168-79.

7. Lonez C, Verma B, Hendlisz A, Aftimos P, Awada A, Van Den Neste E, et al. Study protocol for THINK: a multinational open-label phase I study to assess the safety and clinical activity of multiple administrations of NKR-2 in patients with different metastatic tumour types. BMJ Open. 2017;7(11):e017075.

8. Alexandrov LB, Nik-Zainal S, Wedge DC, Aparicio SA, Behjati S, Biankin AV, et al. Signatures of mutational processes in human cancer. Nature. 2013; 500(7463):415-21.

9. van Loenen MM, de Boer R, Hagedoorn RS, van Egmond EH, Falkenburg JH, Heemskerk MH. Optimization of the HA-1-specific T-cell receptor for gene therapy of hematologic malignancies. Haematologica. 2011;96(3):477-81.

10. Marcu-Malina V, Heijhuurs S, van BM, Hartkamp L, Strand S, Sebestyen Z, et al. Redirecting alphabeta $T$ cells against cancer cells by transfer of a broadly tumor-reactive gammadeltaT-cell receptor. Blood. 2011;118(1):50-9.

11. Gober HJ, Kistowska M, Angman L, Jeno P, Mori L, De LG. Human T cell receptor gammadelta cells recognize endogenous mevalonate metabolites in tumor cells. J Exp Med. 2003;197(2):163-8.

12. Sebestyen Z, Scheper W, Vyborova A, Gu S, Rychnavska Z, Schiffler M, et al. RhoB mediates Phosphoantigen recognition by VgammagVdelta2 $\mathrm{T}$ cell receptor. Cell Rep. 2016;15(9):1973-85.

13. Deniger DC, Moyes JS, Cooper LJ. Clinical applications of gamma delta T cells with multivalent immunity. Front Immunol. 2014;5:636

14. Kunzmann V, Bauer E, Feurle J, Weissinger F, Tony HP, Wilhelm M. Stimulation of gammadelta $\mathrm{T}$ cells by aminobisphosphonates and induction of antiplasma cell activity in multiple myeloma. Blood. 2000;96(2):384-92.

15. Scheper W, Grunder C, Straetemans T, Sebestyen Z, Kuball J. Hunting for clinical translation with innate-like immune cells and their receptors. Leukemia. 2014;28(6):1181-90.

16. Scheper W, Sebestyen Z, Kuball J. Cancer immunotherapy using gammadeltaT cells: dealing with diversity. Front Immunol. 2014;5:601.
17. Straetemans T, Grunder C, Heijhuurs S, Hol S, Slaper-Cortenbach I, Bonig H, et al. Untouched GMP-ready purified engineered immune cells to treat Cancer. Clin Cancer Res. 2015;21(17):3957-68.

18. Grunder C, van DS, Hol S, Drent E, Straetemans T, Heijhuurs S, et al. gamma9 and delta2CDR3 domains regulate functional avidity of T cells harboring gamma9delta2TCRs. Blood. 2012;120(26):5153-62.

19. Gu S, Borowska MT, Boughter CT, Adams EJ. Butyrophilin3A proteins and VgammagVdelta2 T cell activation. Semin Cell Dev Biol. 2018.

20. Gu S, Sachleben JR, Boughter CT, Nawrocka WI, Borowska MT, Tarrasch JT, et al. Phosphoantigen-induced conformational change of butyrophilin 3A1 (BTN3A1) and its implication on Vgamma9Vdelta2 T cell activation. Proc Natl Acad Sci U S A. 2017;114(35):E7311-E20.

21. Harly C, Guillaume Y, Nedellec S, Peigne CM, Monkkonen H, Monkkonen J, et al. Key implication of CD277/butyrophilin-3 (BTN3A) in cellular stress sensing by a major human gammadelta T-cell subset. Blood. 2012;120(11): 2269-79.

22. Palakodeti A, Sandstrom A, Sundaresan L, Harly C, Nedellec S, Olive D, et al. The molecular basis for modulation of human VgammagVdelta2 $\mathrm{T}$ cell responses by CD277/butyrophilin-3 (BTN3A)-specific antibodies. J Biol Chem. 2012;287(39):32780-90.

23. Vavassori S, Kumar A, Wan GS, Ramanjaneyulu GS, Cavallari M, El Daker S, et al. Butyrophilin 3A1 binds phosphorylated antigens and stimulates human gammadelta T cells. Nat Immunol. 2013;14(9):908-16.

24. Braham MVJ, Minnema MC, Aarts T, Sebestyen Z, Straetemans T, Vyborova A, et al. Cellular immunotherapy on primary multiple myeloma expanded in a 3D bone marrow niche model. Oncoimmunology. 2018:e1434465.

25. Wunderlich M, Chou FS, Link KA, Mizukawa B, Perry RL, Carroll M, et al. AML xenograft efficiency is significantly improved in NOD/SCID-IL2RG mice constitutively expressing human SCF, GM-CSF and IL-3. Leukemia. 2010;24(10):1785-8.

26. Gleimer M, Parham P. Stress management: MHC class I and class I-like molecules as reporters of cellular stress. Immunity. 2003;19(4):469-77.

27. Dudley ME, Yang JC, Sherry R, Hughes MS, Royal R, Kammula U, et al. Adoptive cell therapy for patients with metastatic melanoma: evaluation of intensive myeloablative chemoradiation preparative regimens. J Clin Oncol. 2008;26(32):5233-9.

28. Neelapu SS, Tummala S, Kebriaei P, Wierda W, Gutierrez C, Locke FL, et al. Chimeric antigen receptor T-cell therapy - assessment and management of toxicities. Nat Rev Clin Oncol. 2018;15(1):47-62.

29. Linette GP, Stadtmauer EA, Maus MV, Rapoport AP, Levine BL, Emery L, et al. Cardiovascular toxicity and titin cross-reactivity of affinity-enhanced $T$ cells in myeloma and melanoma. Blood. 2013;122(6):863-71.

30. Novosiadly R, Kalos M. High-content molecular profiling of T-cell therapy in oncology. Mol Ther Oncolytics. 2016;3:16009.

31. Xia J, Hu Z, Yoshihara S, Li Y, Jin CH, Tan S, et al. Modeling human leukemia immunotherapy in humanized mice. EBioMedicine. 2016;10:101-8.

32. Barabe F, Kennedy JA, Hope KJ, Dick JE. Modeling the initiation and progression of human acute leukemia in mice. Science. 2007;316(5824):600-4.

33. Lai $Y$, Wei $X$, Lin $S$, Qin L, Cheng L, Li P. Current status and perspectives of patient-derived xenograft models in cancer research. J Hematol Oncol. 2017;10(1):106.

34. Siolas D, Hannon GJ. Patient-derived tumor xenografts: transforming clinical samples into mouse models. Cancer Res. 2013;73(17):5315-9.

35. Sanchez PV, Perry RL, Sarry JE, Perl AE, Murphy K, Swider CR, et al. A robust xenotransplantation model for acute myeloid leukemia. Leukemia. 2009; 23(11):2109-17.

36. Frasca D, Guidi F, Arbitrio M, Pioli C, Poccia F, Cicconi R, et al. Hematopoietic reconstitution after lethal irradiation and bone marrow transplantation: effects of different hematopoietic cytokines on the recovery of thymus, spleen and blood cells. Bone Marrow Transplant. 2000;25(4):427-33.

37. Serafini M, Dylla SJ, Oki M, Heremans Y, Tolar J, Jiang Y, et al. Hematopoietic reconstitution by multipotent adult progenitor cells: precursors to longterm hematopoietic stem cells. J Exp Med. 2007;204(1):129-39.

38. Miller SC. Hematopoietic reconstitution of irradiated, stem cell-injected mice: early dynamics of restoration of the cell lineages of the spleen and bone marrow. J Hematother Stem Cell Res. 2002;11(6):965-70.

39. Gulley JL, Madan RA, Pachynski R, Mulders P, Sheikh NA, Trager J, et al. Role of antigen spread and distinctive characteristics of immunotherapy in Cancer treatment. J Natl Cancer Inst. 2017;109(4).

40. Straetemans T, Kierkels G, Doorn R, Jansen K, Heijhuurs S, dos Santos J, et al. GMP-grade manufacturing of $T$ cells engineered to express a defined YSTCR. Frontier in Immunology. 2018;9:1062. 